\title{
Polyakov conjecture on the supertorus
}

\author{
M. Kachkachi ${ }^{1}$ and M. Nazah ${ }^{2}$ \\ ${ }^{1}$ Département de Mathématiques, F.S.T.S.,B.P. 577, \\ Université Hassan 1 ${ }^{\mathrm{er}}$, Settat, Morocco* \\ and \\ UFR-HEP-Rabat, Université MV, Faculté des Sciences, \\ Département de Physiques, B.P. 1014, Rabat, Morocco \\ 2 Université MV, Faculté des Sciences, \\ Département de Physiques, UFR-Physiques théoriques-Rabat, \\ B.P. 1014, Rabat, Morocco
}

$(10 / 08 / 1997)$

\begin{abstract}
We prove the Polyakov conjecture on the supertorus $\left(S T_{2}\right)$ : we dermine an iterative solution at any order of the superconformal Ward identity and we show that this solution is resumed by the Wess-Zumino-Polyakov (WZP) action that describes the $(1,0) 2 D$-supergavity. The resolution of the superBeltrami equation for the Wess-Zumino (WZ) field is done by using on the one hand the Cauchy kernel defined on $S T_{2}$ in [12] and on the other hand, the formalism developed in [11] to get the general solution on the supercomplex plane. Hence, we determine the n-points Green functions from the (WZP) action expressed in terms of the (WZ) field.
\end{abstract}

*Permanent Address 


\section{INTRODUCTION}

A consistent framework for studying $N=1$ supergravity is provided by the covariant RNS model of the superstring theory where Lorentz invariance is manifest, but space-time supersymmetry is not [1]. In this model superstring theory is formulated as the superfield $\Phi^{\mu}(x, \theta)=X^{\mu}(z)+\theta \Psi^{\mu}(z)$, where $X^{\mu}$ determines the position of the bosonic string and $\Psi^{\mu}$ its supersymmetric partner, coupled to the superzweibein which defines the geometry of the corresponding supergravity theory. However, any supergravity geometry in two dimensions is locally flat which means that there exist local coordinates in which the superzweibein becomes flat. These local complex coordinates together with superconformal transformations define a compact superRiemann surface (SRS) which we denote by $\hat{\Sigma}$. Then, when we consider interactions at a given loop of order $g$, the world sheet of the superstring is $\hat{\Sigma}$. The corresponding action has a large gauge invariance symmetry: it is invariant under

superdiffeomorphisims on $\hat{\Sigma}$, the local supersymmetry whose corresponding gauge field is the gravitino, it is also invariant under superWeyl transformations as well as the local Lorentz transformations of the superzweibein.

In the Polyakov formalism [2], which is geometric and can thus treat global objects, superstring quantization involves functional integration over the superfield $\Phi^{\mu}$ which is gaussian and that over the superzweibein which is non-trivial and leads to two different settings depending on the gauge we choose. In the superconformal gauge obtained after transforming the superzweibein by superdiffeomorphisms and superWeyl rescalings into a flat one, the functional integration analysis leads to the superLiouville theory [3] which represents the degree of freedom of the 2-dimensional supergravity.

One can choose the chiral gauge which has a single non-vanishing metric mode, the superBeltrami differential that represents the graviton-gravitino multiplet, and recast the theory in a local form by introducing the (WZ) field defined by the superBeltrami equation. This field is the projective coordinate that represents the structure parametrized by the superBeltrami differential. Indeed, let us consider a SRS $\hat{\Sigma}$ (without boundary) of genus $g$, with a 
reference conformal structure $\{(z, \theta)\}$ together with an isothermal structure $\{(\hat{Z}, \hat{\Theta})\}$. This is obtained from the reference one by a quasisuperconformal transformation, i.e. a transformation that changes a circle into an ellipse. This transformation is parametrized in general by three superBeltrami differentials of which only two are linearly independent. There is a formalism in which one of the independent differentials is set to zero as it contains only non-physical degrees of freedom, thus ending up with only one superBeltrami differential and this implies the existence of a superconformal structure on the SRS which is necassary for defing the Cauchy-Riemann operator. This formalism is used in $[4,5]$. However, it is more natural from a geometrical point of view to work in another formalism that also reduces the number of superBeltrami differentials to one by eliminating the $\bar{\theta}$-dependence in the coordinate $(\hat{Z}, \hat{\Theta})[6,7]$ in addition to the superconformal structure condition of the previous formalism. The superconformal structure thus defined is parametrized by a single superBeltrami differential $\hat{\mu}$. More importantly, this gauge allows for decoupling the superBeltrami equations satisfied by $\hat{Z}$ and by the (WZ) field $\hat{\Theta}$ and then are more easly solved using the techniques of the Cauchy kernel. The solutions thus obtained enable us to write the action as a functional of the superBeltrami differential $\hat{\mu}$ from which we compute the Green functions and the energy-momentum tensor whose external source is $\hat{\mu}$.

In this parametrization the superWeyl invariant effective action splits into two terms, i.e.

$$
\Gamma\left[\hat{\mu}, \overline{\hat{\mu}} ; \hat{R}_{0}, \overline{\hat{R}}_{0}\right]=\Gamma_{W Z P}\left[\hat{\mu} ; \hat{R}_{0}\right]+\bar{\Gamma}_{W Z P}\left[\overline{\hat{\mu}} ; \overline{\hat{R}}_{0}\right]
$$

where $\hat{R}_{0}$ is a holomorphic background superprojective connection in the superconformal structure $\{(z, \theta)\}$, i.e. $\bar{D}_{\theta} \hat{R}_{0}=0$, which is introduced to insure a good glueing of the anomaly on $\hat{\Sigma}$. $\Gamma_{W Z P}$ is the Wess-Zumino-Polyakov action which describes the 2D induced quantum supergravity in the light-cone gauge, i.e. $d s^{2}=(d z+\hat{\mu} d \bar{z}+\theta d \theta) d \bar{z}$. It depends on the background conformal geometry parametrized by the pair $\left(\hat{\mu}, \hat{R}_{0}\right)$ and satisfies the superconformal Ward identity [8,9]

$$
\left(\bar{\partial}-\hat{\mu} \partial-\frac{3}{2} \partial \hat{\mu}-\frac{1}{2} D \hat{\mu} D\right) \frac{\delta \Gamma_{W Z P}}{\delta \hat{\mu}}=k \partial^{2} D \hat{\mu},
$$


where $\mathrm{k}$ is the central charge of the model which is the remnant of the matter system after functional integration. It measures the strength of the superdiffeomorphisms anomaly.

Solving eq.(1.2) on a superRiemann surface of genus $g$ is the starting point for studying 2dimensional superconformal models theron. A solution to this superconformal Ward identity was found by Grundberg and Nakayama in [10] on the supercomplex plane. Then, the Polyakov conjecture on the supercomplex plane, which tels us that the iterative solution of eq.(1.2) is resumed by the (WZP) action, is proved in [11]. The generalization of this solution, at the third order in perturbative series in terms of $\hat{\mu}$, to the supertorus was given in [12] and that to a $g$-SRS was performed in [5]. The subject of this work is to prove the Polyakov conjecture on the supertorus $S T_{2}$ at any order of the perturbative series and then to compute the $n$-points Green function for generic $n$ starting from the (WZP) on $S T_{2}$.

To do this, we consider on the one hand the superquasielliptic Weierstrass $\hat{\zeta}$-function (the supersymmetric extension of the Weierstrass $\zeta$-function) constructed in [12] as the $\bar{\partial}$-Cauchy kernel on $S T_{2}$ to solve the superBeltrami equations (SBE). On the other hand, we adopt here the formalism developed in [11] to get the perturbative series solution on the supercomplex plane.

\section{RESOLUTION OF (SBE) ON $S T_{2}$}

The superBeltrami equation in terms of the (WZ) field $\hat{\Theta}$ can be writen as $[11,12]$ :

$$
\bar{\partial} \Lambda=\frac{1}{2} \partial \hat{\mu}+B D \Lambda
$$

where $B=\hat{\mu} D+\frac{1}{2} D \hat{\mu}, \Lambda=\ln D \hat{\Theta}$ and $D=\partial_{\theta}+\theta \partial_{z}$. Then, using the generalized Cauchy formula introduced in [12] that is,

$$
\left(\bar{\partial}^{-1} F\right)\left(z_{1}, \theta_{1}\right)=\int_{S T_{2}} d \tau_{2} \hat{\zeta}_{1,2} F\left(z_{2}, \theta_{2}\right),
$$

where

$$
\hat{\zeta}_{1,2} \equiv\left(\theta_{2}-\theta_{1}\right) \zeta\left(z_{1}-z_{2}\right)
$$




$$
d \tau_{2} \equiv \frac{d z_{2} \wedge d \bar{z}_{2}}{2 \pi i} d \theta_{2}
$$

and

$$
\int_{S T_{2}} d \tau_{2} \delta^{3}\left(a_{1}-a_{2}\right) F\left(a_{2}\right)=F\left(a_{1}\right)
$$

with

$$
a_{i} \equiv\left(z_{i}, \bar{z}_{i}, \theta_{i}\right)
$$

we get the solution of eq.(2.1) as a formal series:

$$
\Lambda=\sum_{n=1}^{\infty} \bar{\partial}^{-1} \lambda_{n}(z, \theta)
$$

with

$\lambda_{1}=\frac{1}{2} \partial \hat{\mu}$ and $\lambda_{n}=B D \bar{\partial}^{-1} \lambda_{n-1}$.

For $n \geq 2$ we find, for the $n$-term of the series (2.6) the expression

$$
\bar{\partial}^{-1} \lambda_{n}=(-1)^{\frac{n(n-1)}{2}} \int_{S T_{2}} \prod_{j=2}^{n+1} d \tau_{j} \prod_{i=1}^{n-1}\left(\hat{\zeta}_{i, i+1} B_{i+1} D_{i+1}\right) \hat{\zeta}_{n, n+1} \lambda_{1}\left(a_{n+1}\right) .
$$

$B_{i}$ means that $B$ is evaluated at the point $a_{i}$. The sign in front of the integral arises from the commuation of the Cauchy kernel $\hat{\zeta}$ with the product of measures $\prod_{j=2}^{n+1} d \tau_{j}$. Here we have adopted the convention $\prod_{i=1}^{0}\left(\hat{\zeta}_{i, i+1} B_{i} D_{i+1}\right) \equiv 1$. One should note that the formula $(2.7)$ contains a power of the superBeltrami differential $\hat{\mu}$ and its derivatives. In order to express this equation in powers of $\hat{\mu}$ only, we rewrite eq.(2.7) as follows:

$$
\begin{gathered}
\bar{\partial}^{-1} \lambda_{n}\left(a_{1}\right)=(-1)^{\frac{n(n-1)}{2}} \int_{S T_{2}} \prod_{j=2}^{n+1} d \tau_{j} f_{1, k-1} \hat{\zeta}_{k, k+1} \partial_{k+1} f_{k+1, n-1} . g+ \\
(-1)^{\frac{n(n-1)}{2}} \int_{S T_{2}} \prod_{j=2}^{n+1} d \tau_{j} f_{1, k-1} \hat{\zeta}_{k, k+1}\left(D_{k+1} \hat{\mu}\left(a_{k+1}\right)\right) D_{k+1} f_{k+1, n-1} . g,
\end{gathered}
$$

where $f_{l, m}=\prod_{i=l}^{m}\left(\hat{\zeta}_{i, i+1} B_{i+1} D_{i+1}\right), g=\hat{\zeta}_{n, n+1} \lambda_{1}\left(a_{n+1}\right)$ and where the $k$-term of the product $\left(\hat{\zeta}_{k, k+1} B_{k+1} D_{k+1}\right)$ was developed.

The integration by parts of the second term in the r.h.s.l of eq.(2.8) yields 


$$
\bar{\partial}^{-1} \lambda_{n}\left(a_{1}\right)=\frac{(-1)^{\frac{n(n-1)}{2}}}{2^{n}} \int_{S T_{2}} \prod_{j=2}^{n+1} d \tau_{j}\left[\prod_{i=1}^{n-1}\left(\hat{\zeta}_{i, i+1} \partial_{i+1}-D_{i} \hat{\zeta}_{i, i+1} D_{i+1}\right) \partial_{n} \hat{\zeta}_{n, n+1} \prod_{l=2}^{n+1}\left(a_{l}\right)\right]
$$

and then, the sommation over the index $n$ gives the superfield $\Lambda$.

For example, one can verify that $\Lambda$ is given at the second order in $\hat{\mu}$ by the relation

$$
\Lambda\left(a_{1}\right)=\frac{1}{2} \int_{S T_{2}} d \tau_{2} \partial_{1} \hat{\zeta}_{1,2} \hat{\mu}\left(a_{2}\right)-\frac{1}{4} \int_{S T_{2}} d \tau_{23}\left[\left(\hat{\zeta}_{1,2} \partial_{2}-D_{1} \hat{\zeta}_{1,2} D_{2}\right) \partial_{2} \hat{\zeta}_{2,3}\right] \hat{\mu}\left(a_{2}\right) \hat{\mu}\left(a_{3}\right)
$$

where $d \tau_{23} \equiv d \tau_{2} d \tau_{3}$, that agrees with the solution given in [12].

Hence, we have obtained the perturbative expression for the superprojective coordinates $(\hat{Z}, \hat{\Theta})$ in terms of the reference complex structure $(z, \bar{z}, \theta)$ on the supertorus.

\section{THE $N$-POINTS GREEN FUNCTION FROM THE WZP ACTION ON $S T_{2}$}

The WZP action on the supertorus $S T_{2}$ introduced in [5] is expressed as

$$
\Gamma_{W Z P}\left[\hat{\mu}, R_{0}\right]=k \int_{S T_{2}} d \tau_{1}\left\{2\left(R_{0}-R\right) \hat{\mu}+\left(\chi-\chi_{0}\right) \Delta_{\chi} \hat{\mu}\right\}\left(a_{1}\right)
$$

where $\chi=-D \ln D \hat{\Theta}$ is a superaffine connection, $\Delta_{\chi} \hat{\mu} \equiv(\partial-2 D \chi+\chi D) \hat{\mu}$. $R_{0}$ is the

background superprojective connection introduced to guarantee the global definition of the anomaly on $S T_{2}$. The superaffine connection $\chi_{0}$ appears in the action (3.1) to make it globally defined. However, this does not enter the superdiffeomorphims anomaly because it is not a fundamental parameter in the theory and does not contribute to the stress-energy tensor whose exterior source is $\hat{\mu}$ :

$$
T\left(a_{1}\right)=2 k\left(R_{0}-R\right)
$$

$R=-\partial \chi-\chi D \chi$ is the superprojective connection. After some manipulation by considering the anti-commuting property of $\chi$, the action (3.1) reduces to the expression

$$
\Gamma_{W Z P}\left[\hat{\mu}, R_{0}\right]=F\left[\chi_{0}, \partial \Lambda, \hat{\mu}, D \hat{\mu}, R_{0}\right]+k \int_{S T_{2}} d \tau_{1} \partial_{1} D_{1} \Lambda\left(a_{1}\right) \hat{\mu}\left(a_{1}\right)
$$

where $F$ is some functional that does not contribute to the $n$-points Green function for $n \geq 2$.

Nows, eqs.(2.6) and (2.9) enable us to express the action (3.3) in the following form: 


$$
\begin{gathered}
\Gamma_{W Z P}=F+k \pi \sum_{n=1}^{\infty} \frac{(-1)^{\frac{n(n+1)}{2}}}{2^{n}} \int_{S T_{2}} \prod_{j=1}^{n+1} d \tau_{j}\left[\partial _ { 1 } D _ { 1 } \prod _ { i = 1 } ^ { n + 1 } \left(\hat{\zeta}_{i, i+1} \partial_{i+1}\right.\right. \\
\left.\left.-D_{i} \hat{\zeta}_{i, i+1} D_{i+1}\right) \partial_{n} \hat{\zeta}_{n, n+1}\right] \prod_{l=1}^{n+1} \hat{\mu}(l) .
\end{gathered}
$$

Then, from this action, we derive the $n$-points Green function as follows:

$$
\begin{gathered}
<T(1) \ldots T(n)>\left.\equiv(-1)^{n} \frac{\delta^{n} \Gamma_{W Z P}}{\delta \hat{\mu}(1) \ldots \delta \hat{\mu}(n)}\right|_{\hat{\mu}(n)=0} \\
=k \frac{(-1)^{\frac{n(n-1)}{2}}}{(2 \pi)^{n-1}} \sum_{p e r m(p \neq 1)}(-1)^{p} \partial_{1} D_{1} \prod_{i=1}^{n-2}\left(\hat{\zeta}_{i, i+1} \partial_{i+1}-D_{i} \hat{\zeta}_{i, i+1} D_{i+1}\right) \partial_{n-1} \hat{\zeta}_{n-1, n} .
\end{gathered}
$$

The sum over all possible permutations, except for $p \neq 1$, is inderstood and $(-1)^{p}$ stands for the sign of the permutation. Furthermore, after some algebraic calculations, we get the final expression for the $n$-points Green function of the induced (1,0)-supergravity on the supertorus:

$$
\begin{gathered}
<T(1) \ldots T(n)>=\frac{k(-1)^{\frac{n(n+1)}{2}}}{2(2 \pi)^{n-1}} \sum_{p e r m(p \neq 1)}(-1)^{p}\left[\prod _ { i = 1 } ^ { n - 2 } \left(2 \hat{\zeta}_{i, i+1} \partial_{i+1}+D_{i} \hat{\zeta}_{i, i+1} D_{i+1}-\right.\right. \\
\left.3 \partial_{i} \hat{\zeta}_{i, i+1}\right) \partial_{n-1}^{2} \hat{\zeta}_{n-1, n} .
\end{gathered}
$$

Then, we derive the corresponding Ward identitty to the $n$-points Green function by applying the Cauchy operator defined say, at a point $a_{1}$, on the l.h.s of eq.(3.6):

$$
\begin{gathered}
\bar{\partial}_{1}<T(1) \ldots T(n)>=\frac{k(-1)^{\frac{n(n+1)}{2}}}{2^{n}} \sum_{p e r m(p \neq 1)}(-1)^{p}\left(2 \delta^{3}\left(a_{1}-a_{2}\right) \partial_{2}+D_{1} \delta^{3}\left(a_{1}-a_{2}\right) D_{2}-\right. \\
\left.3 \partial_{1} \delta^{3}\left(a_{1}-a_{2}\right)\right) \prod_{i=2}^{n-2}\left(2 \hat{\zeta}_{i, i+1} \partial_{i+1}+D_{i} \hat{\zeta}_{i, i+1} D_{i+1}-3 \partial_{i} \hat{\zeta}_{i, i+1}\right) D_{n-1} \partial_{n-1}^{2} \hat{\zeta}_{n-1,1}
\end{gathered}
$$

For example, putting $n=3$ in eqs.(3.6) and (3.7) we recover the results established in [12] for the 3-points function and its associated Ward identity that are respectively, 


$$
\begin{gathered}
<T(1) T(2) T(3)>=\frac{k}{2(2 \pi)^{2}} \sum_{\text {perm }(p \neq 1)}(-1)^{n}\left(2 \hat{\zeta}_{1,2} \partial_{2}+D_{1} \hat{\zeta}_{1,2} D_{2}-3 \partial_{1} \hat{\zeta}_{1,2}\right) D_{2} \partial_{2}^{2} \hat{\zeta}_{2,3}, \\
\bar{\partial}_{1}<T(1) T(2) T(3)>=\frac{k}{2(2 \pi)^{2}}\left\{\left[2 \delta^{3}\left(a_{1}-a_{2}\right) \partial_{2}+\right.\right. \\
\left.\left.D_{1} \delta^{3}\left(a_{1}-a_{2}\right) D_{2}-3 \partial_{1} \delta^{3}\left(a_{1}-a_{2}\right)\right] D_{2} \partial_{2}^{2} \hat{\zeta}_{2,3}-(2 \leftrightarrow 3)\right\} .
\end{gathered}
$$

This shows that our formalism developed in [11] is general and applicable for any SRS of genus $g$.

\section{SOLUTION OF THE SUPERCONFORMAL WARD IDENTITY ON $S T_{2}$}

Now, let us rewrite the superconformal Ward identitty (1.2) in the form:

$$
\bar{\partial}\left(\frac{\delta \Gamma_{W Z P}}{\delta \hat{\mu}}\right)=p_{1}+K \frac{\delta \Gamma_{W Z P}}{\delta \hat{\mu}}
$$

with $p_{1}=k \partial^{2} D \hat{\mu}$ and $K=\hat{\mu} \partial+\frac{3}{2} \partial \hat{\mu}+\frac{1}{2} D \hat{\mu} D$.

Then, using the iterative method given in section 2 we get

$$
\frac{\delta \Gamma_{w z p}}{\delta \hat{\mu}}=\sum_{n=1}^{\infty} \bar{\partial}^{-1} p_{n}
$$

where $p_{n}=K \bar{\partial}^{-1} p_{n-1}$

and

$$
\begin{gathered}
\bar{\partial}^{-1} p_{n}=\frac{(-1)^{\frac{n(n-1)}{2}}}{2^{n-2}} k \int_{S T_{2}} \prod_{j=2}^{n+1} d \tau_{j} \hat{\zeta}_{1,2} \prod_{i=2}^{n-1}\left[\hat{\mu}(i) \hat{\zeta}_{i, i+1} \partial_{i+1}+\frac{3}{2} \partial_{i} \hat{\mu}(i) \hat{\zeta}_{i, i+1}\right. \\
\left.+\frac{1}{2} D_{i+1} \hat{\mu}(i) D_{i} \hat{\zeta}_{i, i+1} \partial_{n+1}^{2} D_{n+1} \hat{\mu}(n+1)\right]
\end{gathered}
$$

Furthermore, to express eq.(4.3) in terms of $\hat{\mu}$ only, the integration by parts must be considered and then, we obtain

$$
\bar{\partial}^{-1} p_{n}=\frac{(-1)^{\frac{n(n+1)}{2}}}{2^{n-1}} k \int_{S T_{2}} \prod_{j=2}^{n+1} d \tau_{j} \prod_{i=1}^{n-1}\left[\left(2 \hat{\zeta}_{i, i+1} \partial_{i+1}\right.\right.
$$




$$
\left.\left.+D_{i} \hat{\zeta}_{i, i+1} D_{i+1}-3 \partial_{i} \hat{\zeta}_{i, i+1}\right) D_{n} \partial_{n}^{2} \hat{\zeta}_{n, n+1} \prod_{l=2}^{n+1} \hat{\mu}(l)\right]
$$

Hence, by using eq.(4.2) we obtain $\frac{\delta \Gamma_{W Z P}}{\delta \hat{\mu}(1)}$ and the integration of the later gives the $n$-points Green function which coincides with eq.(3.6). Furthermore, this result means that the Polyakov action is the sum of the perturbative series that is a solution of the superconformal Ward identity and then the Polyakov conjecture on the supertorus is proved.

\section{CONCLUSION AND OPEN PROBLEMS}

In this paper we have proved the Polyakov conjecture on the supertorus by using on the one hand the solution of the (SBE) established with the help of the superWeiestrass $\hat{\zeta}$-fuction introduced in [12] and, on the other hand the material developed in [11] to get the general ( $n$-points) Green function on the supercomplex plane.

However, one can express the superLiouville theory in the framework of the formalism developed here and in the refs.[11,12] by considering the superconformal gauge. This can be done by expressing the Liouville field in terms of the Beltrami field $\mu$. This Liouville field can be seen to verify the classical Liouville equation [13]

$$
-\Delta \Psi=R-\exp (-2 \Psi) R_{0}
$$

by taking a conformally equivalent metric to a given metric of constant curvature (i.e.g $=$ $\left.\exp (2 \Psi) g_{0}\right)$. Then, the supersymmetric extension of this development can be easly established.

\section{ACNOWLEDGMENT}

One of the authors (M.K.) would like to thank Professor M. Virasoro for his hospitality at ICTP where this work was partially done. 


\section{REFERENCES}

[1] M. Kaku, Introduction to superstrings, Berlin, Heidelberg, New York, Springer 1988.

[2] A. M. Polyakov, Phys.Lett. 103 B (1981) 207; Phys. Lett. 103 B (1981) 211.

[3] J. Distler and H. Kawi, Nucl. Phys. B321 (1989) 509.

[4] J. -P. Ader and H. Kachkachi, Class. Quantum Grav. 10 (1993) 417.

[5] J. -P. Ader and H.Kachkachi, Class. Quantum Grav. 11 (1994) 767.

[6] L. Crane and J. Rabin, Commun. Math. Phys. 113 (1988) 601.

[7] M. Takama, Commun. Math. Phys. 143 (1991) 149.

[8] M. T. Grisaru and R. -M. Xu, Phys. Lett. B205 (1993) 1.

[9] F. Delduc and F. Gieres, Class. Quantum Grav. 7 (1990) 1907.

[10] J. Grundberg and R. Nakayama, Mod. Phys. Lett. A4 (1989) 55.

[11] M. Kachkachi and S. Kouadik, J. Math. Phys. 38(7) (1997).

[12] H. Kachkachi and M. Kachkachi, Class. Quantum Grav. 11 (1994) 493.

[13] C. Itzykson and J. -M. Drouffe, Statistical field theory: 2, Cambridge University Press, Cambridge 1989. 